olfactory bulb may reflect physicochemical mixture features, a computational analysis of a large odorant-induced imaging database suggested that perceptual categories may also be evident in patterns of glomerular activation ${ }^{9}$. Thus, olfactory bulb representations, and their apparent susceptibility to modification through experience, are likely to be highly influenced by the method of neural recording.

The primary component of the study by Chapuis and Wilson ${ }^{1}$, however, is a direct and powerful test of cortical representation. Behavioral studies in humans have implicated cortical mechanisms is adult odor learning ${ }^{10}$, but which cortical structures were involved was unknown. Although Chapuis and Wilson's results ${ }^{1}$ combine with an additional study in rats ${ }^{11}$ to imply olfactory object representation in APC, a multivariate functional magnetic resonance imaging (fMRI) study in humans implied such olfactory object representation in posterior piriform cortex (PPC), but not in $\mathrm{APC}^{12}$. Moreover, human imaging studies have suggested that APC reflects expectations related to the odorant, more than the odorant itself ${ }^{13,14}$. These differences across studies may reflect the difference in species (rat versus human), method (single-unit recording versus fMRI), state of consciousness (anesthesia versus wake) or task (positive reinforcement in rats versus aversive learning in humans). Regardless of these potential differences across PPC and APC, Chapuis and Wilson's results ${ }^{1}$ indicate that piriform cortex representations are reflected in highly malleable learning-dependent patterns of activity. Such ongoing rapid malleability in the adult brain is inconsistent with the typical view of primary cortical representations, suggesting that the structures classically referred to as primary olfactory cortex may not be primary at all.

\section{COMPETING FINANCIAL INTERESTS}

The authors declare no competing financial interests.

1. Chapuis, J. \& Wilson, D.A. Nat. Neurosci. 15, 155-161 (2011).

2. Carmichael, S.T., Clugnet, M.C. \& Price, J.L. J. Comp Neurol. 346, 403-434 (1994).

3. Haberly, L.B. Chem. Senses 26, 551-576 (2001).

4. Barnes, D.C., Hofacer, R.D., Zaman, A.R., Rennaker, R.L. \& Wilson, D.A. Nat. Neurosci. 11, 1378-1380 (2008).

5. Wilson, D.A., Sullivan, R.M. \& Leon, M. J. Neurosci. 7, 3154-3162 (1987)

6. Kay, L.M. \& Laurent, G. Nat. Neurosci. 2, 1003-1009 (1999).

7. Lin, D.Y. Neuron 50, 937-949 (2006).

8. Johnson, B.A., Ong, J. \& Leon, M. J. Comp. Neurol. 518 1542-1555 (2010)

9. Auffarth, B., Gutierrez-Galvez, A. \& Marco, S. Front Syst. Neurosci. 5, 82 (2011)

10. Mainland, J.D. et al. Nature 419, 802 (2002).

11. Kadohisa, M. \& Wilson, D.A. Proc. Natl. Acad. Sci. USA 103, 15206-15211 (2006).

12. Li, W., Howard, J.D., Parrish, T.B. \& Gottfried, J.A. Science 319, 1842-1845 (2008).

13. Zelano, C., Mohanty, A. \& Gottfried, J.A. Neuron 72 178-187 (2011)

14. Zelano, C. et al. Nat. Neurosci. 8, 114-120 (2005).

\title{
Targets of your daily CA(2)ppuccino
}

Caffeine is believed to be the world's most widely used psychoactive stimulant and if you've ever been stuck in a long queue at your local Starbucks, it would be difficult to argue. For many, daily caffeine intake, usually in the form of one or more cups of coffee, serves to increase alertness and mental acuity; however, the precise cellular and molecular mechanisms that mediate these effects are not clear.

Caffeine is an antagonist of the purinergic, $G$ protein-coupled $A_{1}$ adenosine receptor $\left(A_{1} R\right)$ and previous studies have suggested that inhibition of the $A_{1} R$ in the CA1 region of the hippocampus can induce an enhancement of long-term potentiation. However, it is the less well-characterized CA2 region that contains the highest concentrations of these adenosine-activated receptors and thus represents a potential target for caffeine. Similar to the adjacent $\mathrm{CA} 1$, the $\mathrm{CA} 2$ region receives specialized inputs from the CA3, which are known as Schaffer collaterals. Unlike those that project to the CA1, however, these inputs do not appear to exhibit activity-dependent potentiation, suggesting that, if caffeine does modulate synaptic plasticity, it would do so via a different mechanism. In a Brief Communication on page 24, Simons and colleagues show that physiological doses of caffeine can selectively modulate synaptic transmission in the CA2 region of the hippocampus via a mechanism that is independent of the traditional NMDA/Ca ${ }^{2+}$-mediated plasticity.

The authors found that if they fed juvenile rats with physiological doses of caffeine and, $1 \mathrm{~h}$ later, recorded from hippocampal tissue slices, there was a significant increase in the amplitude of excitatory postsynaptic currents in the CA2, but not in the CA1,

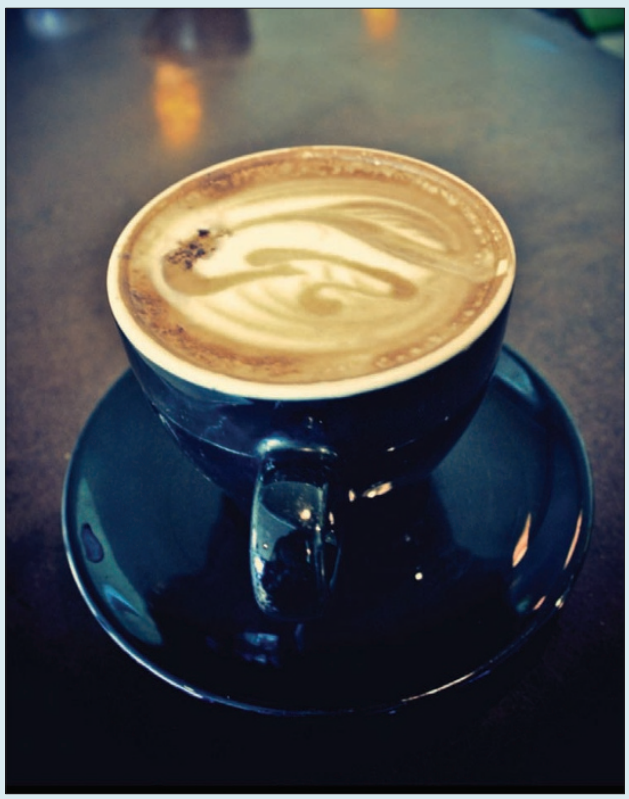
region and that this effect did not result from a change in neuronal excitability. They found the same effect when caffeine was added directly to naive hippocampal slices, further supporting the specificity of this effect. This change in synaptic responsiveness persisted for at least $3 \mathrm{~h}$.

In the CA1 region, induction of long-term potentiation is dependent on NMDA receptor activation and consequent $\mathrm{Ca}^{2+}$-dependent signaling. However, the authors found that the $\mathrm{A}_{1} \mathrm{R}$-mediated CA2 potentiation was not perturbed by a calcium chelator, an NMDA receptor antagonist or inhibitors of calcium signaling. Instead, this synaptic plasticity was potently blocked by the addition of pharmacological inhibitors of either adenylyl cyclase or protein kinase $\mathrm{A}$, which is consistent with the fact that the $\mathrm{A}_{1}$ Rs are known to be coupled to the adenylyl cyclase-modulating $\mathrm{G}_{\mathrm{i} / \mathrm{o}}$ proteins. In addition, blockade of the MAPK signaling pathway also appeared to mitigate the stabilization of the $\mathrm{A}_{1} \mathrm{R}$-dependent plasticity, hinting at a more complex signaling mechanism governing the consolidation of this synaptic plasticity.

Although it remains unclear whether the burst of mental clarity you receive from your morning coffee is a direct result of caffeine inhibiting adenosine receptors in the CA2 region of the hippocampus, this report provides a good starting point to unravel the mechanisms by which caffeine stimulates the brain.

Timothy Spencer 